\title{
Numerical Simulation of an Active Feedback Control of Sheet Flutter in a Narrow Passage*
}

\author{
Masahiro WATANABE $^{* *}$ and Yutaka KOYAMA ${ }^{* *}$ \\ ** Department of Mechanical Engineering, Aoyama Gakuin University \\ 5-10-1 Fuchinobe, Sagamihara-shi, Kanagawa 229-8558, Japan \\ E-mail: watanabe@me.aoyama.ac.jp
}

\begin{abstract}
This paper deals with numerical simulations of an active feedback control of leakage-flow-induced sheet flutter in a narrow passage. In this paper, a new non-contact control device and active feedback control technique by using moving baffle-plates to suppress the sheet flutter are proposed and its numerical model is developed by using finite-segment method based on multi-body dynamics formulation. The control performance of the proposed control device and active feedback control method is evaluated by the numerical simulations. In the modeling of the system, the flexible sheet consists of some rigid bodies connected with rotational springs and dampers based on multi-body dynamics formulation. The nonlinear fluid forces acting on the rigid bodies are derived from the basic equations of leakage fluid flow in a narrow passage. The nonlinear equation of motion of the flexible sheet coupled with the fluid flow controlled by the moving baffle-plate actuators is calculated numerically. By the numerical simulations, it is shown that sheet flutter is effectively suppressed by the proposed non-contact control device and active feedback control. Moreover, the control performance is evaluated with changing controller gain and phase shift angle of the control signal.
\end{abstract}

Key words: Sheet Flutter, Active Feedback Control, Control Device, Multi-Body Dynamics, Nonlinear Dynamics, Numerical Simulation.

\section{Introduction}

Leakage-flow-induced sheet flutter is a self-excited vibration generated by the fluid-structure interaction between out-of-plane motion of flexible sheet and leakage fluid flow in a narrow passage, and is often encountered in many engineering applications, such as high-speed copy machines. The sheet flutter reduce the process efficiency and generate noise, in some case, cause fatigue failure to the flexible thin sheet moving at a high speed. Moreover, in this system, it is difficult to increase the structural damping of the thin sheet and to add a contact force directly to the flexible sheet moving at a high speed in order to suppress the sheet flutter. Thus, we need a non-contact vibration control technique and new control device to suppress the sheet flutter generated in the flexible thin sheet moving at a high speed.

Up to this time, many studies on the stability of the leakage-flow-induced vibrations and sheet flutter have been reported in Refs. (1) (5). Inada and Hayama, in Ref.(1), studied leakage-flow-induced vibrations of translational one-degree-of-freedom system and clarified instability mechanism theoretically. From these studies, it was found that self-excited vibrations occur due to negative fluid damping when a narrow passage is divergent downstream or it includes a constriction at the inlet or a diffuser at the outlet. 
Nagakura and Kaneko, in Ref.(2), studied the stability of a flexible beam subjected to fluid flow in a narrow passage. Yamaguchi and co-authors, in Refs. (3) and (4), studied the dynamic behavior and stability of a flexible thin sheet subjected high-speed fluid flow. Kaneko and Wu, in Ref.(5), developed a nonlinear numerical model of a sheet flutter based on multi-body dynamics formulation and examined the nonlinear dynamic behavior and stability of the sheet flutter.

Some studies, in Refs. (6) (15), on the active control of flow-induced vibrations such as vortex-induced vibrations, flow oscillations in jet-wedge and cavity systems, compressor surge, and leakage-induced vibrations have been reported. Kaneko and Hirota, in Ref.(14), studied the active control of a leakage flow induced vibration which occurs to a rigid plate elastically supported in a narrow tapered passage. In this study, a bimorph-type piezoelectric actuator attached at the upstream edge of the plate was used to generate an added fluid force as a control force. In our previous paper, in Ref.(15), we proposed a new non-contact active feedback control of leakage-flow-induced vibrations in a narrow passage, by using the injection and suction of fluid at inlet or outlet of the passage, and we applied this active control technique to suppress a leakage-flow-induced vibration in translational one-degree-of-freedom system.

In this paper, we propose a new non-contact control device and active feedback control technique by using moving baffle-plates to suppress the leakage-flow-induced sheet flutter, and develop its numerical model based on multi-body dynamics formulation. The fluid flow in the narrow passage is actively controlled by the motion of the baffle-plate actuators, which are set at the inlet of the passage. The strategy of the proposed non-contact control device and active feedback control technique is based on the concept of active flow control. The motion of the baffle-plate actuator controls the fluid flow to remove the original source of the exciting fluid force caused by the fluid-structure interaction, i.e., cancels the self-excited feedback mechanism. In the numerical simulations, not only nonlinear structural motion of the flexible sheet but also nonlinear fluid force acting on the flexible sheet is considered. The nonlinearity of the structural motion of the flexible sheet are considered based on multi-body dynamics formulation, and the nonlinear fluid force acting on the flexible sheet are given by the nonlinear numerical model developed by Kaneko and $\mathrm{Wu}$, in Ref.(5). Moreover, nonlinear control effect of the baffle-plate actuator on the fluid flow is considered. By numerical simulations, it is shown that leakage-flow-induced sheet flutter is effectively suppressed by the proposed control device and active feedback control technique. Moreover, the control performance is evaluated with changing controller gain and phase shift of the control signal against the sensor signal.

\section{Nomenclature}

$\begin{array}{lll}c_{\varphi} & : & \text { Damping coefficient of damper connecting body } i \text { and body } i+1 \\ D & : & \text { Passage length } \\ F & : & \text { Fluid force acting on rigid body element } \\ G^{*} & : & \text { Controller gain of control signal } \\ h & : & \text { Gap width of passage } \\ J & : & \text { Moment of inertia of rigid body element } \\ k_{\varphi} & : & \text { Stiffness of rotational spring connecting body } i \text { and body } i+1 \\ 2 \ell & : & \text { Length of rigid body element } \\ L & : & \text { Sheet length } \\ m & : & \text { Mass of rigid body element } \\ n & : & \text { Number of rigid body element } \\ P & : & \text { Fluid Pressure in the passage } \\ P_{a}, P_{b} & : \text { Fluid pressure at inlet and outlet of the passage, respectively } \\ Q_{f} & : \text { Flow rate } \\ t & : \text { Time }\end{array}$




$\begin{array}{ll}t_{s} & : \text { Thickness of sheet } \\ U & : \text { Flow velocity } \\ u_{h} & : \text { Output displacement of the actuator ( moving baffle-plate ) } \\ v_{h} & : \text { Control signal } \\ w & : \text { Vibration displacement at sensor position } \\ w_{r m s}, w_{r m s-c} & : \text { RMS value of } w \text { without and with control, respectively } \\ \lambda_{f} & : \text { Friction coefficient of passage wall } \\ v & : \text { Kinematic viscosity of fluid } \\ \xi_{i n}, \xi_{e x} & : \text { Pressure loss coefficient at inlet and outlet of the passage, respectively } \\ \rho_{f} & : \text { Fluid density } \\ \phi & : \text { Phase shift angle of control signal } \\ \varphi_{i} & : \text { Rotational angle between body } i \text { and body } i+1 \\ o-x y & : \text { Global coordinate system } \\ o-x^{\prime} y^{\prime} & : \text { Body coordinate system }\end{array}$

\section{Numerical Model and Active Feedback Control Method}

The numerical model of the sheet flutter with the active feedback control is shown in Fig.1. A flexible sheet is set in the middle of a narrow passage, and is clamped at upstream end and free at downstream end. The flexible sheet is subjected to fluid flow in the narrow passage. When the flow velocity becomes higher, the leakage-flow-induced sheet flutter occurs to the flexible sheet due to the fluid flow in the passage.
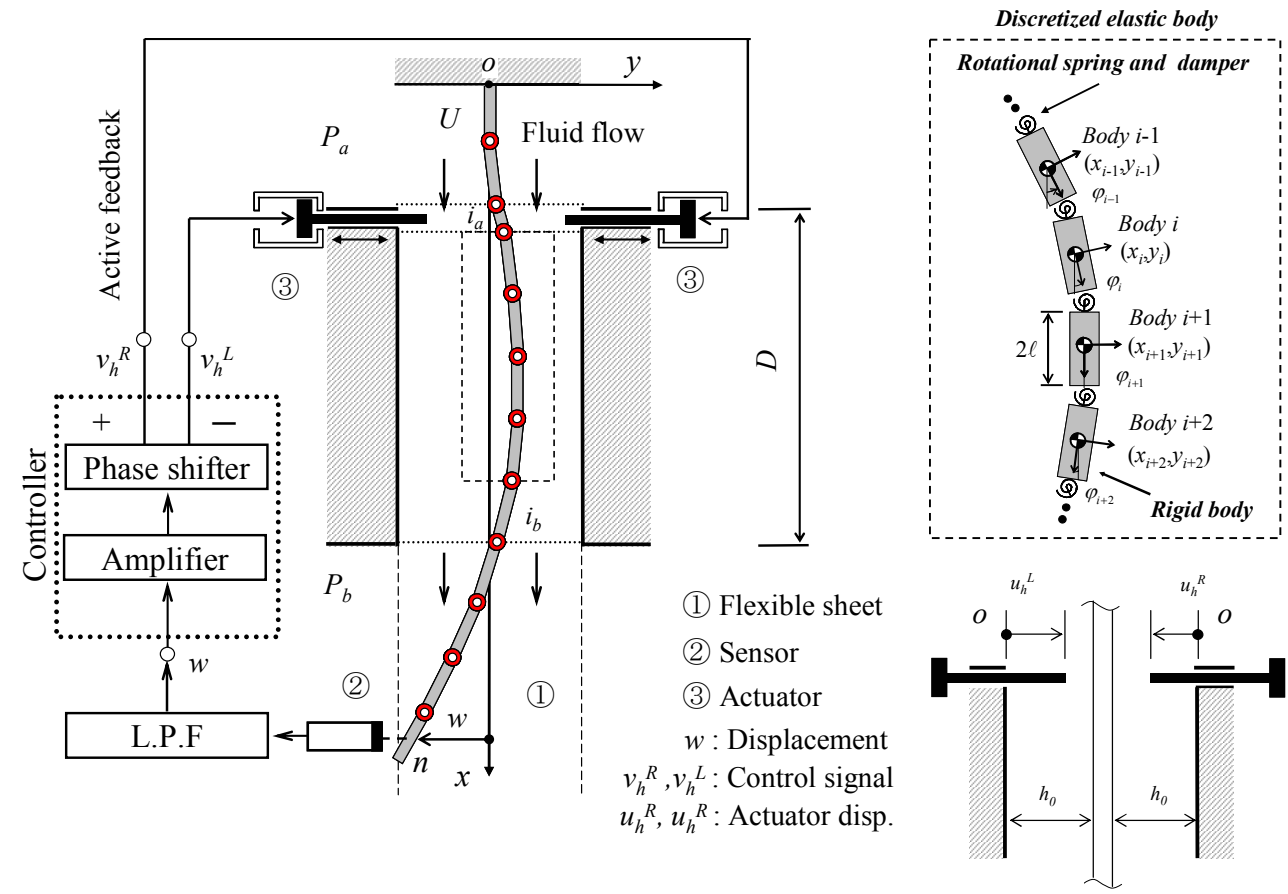

Fig.1 Numerical model and signal flow of active feedback control

In the active feedback control, the sheet flutter is suppressed by the active flow control, in which the fluid flow in the passage is controlled by the moving baffle-plate actuators set at the inlet of the passage. The motions of the baffle plate actuators on both side of the sheet are driven by the control signal $v_{h}^{R}$ and $v_{h}^{L}\left(=-v_{h}^{R}\right)$ from a controller which consists of an amplifier and phase shifter. The output displacements of the baffle plates are defined as $u_{h}^{R}$ 
and $u_{h}^{L}\left(=-u_{h}^{R}\right)$, and are made by two linear actuators using a sensor signal $w$ with amplifier gain $G^{*}$ and phase shift $\phi$. The sensor signal $w$ of the vibration displacement at the tip of the sheet is obtained by a sensor through a pre-amplifier and a low-pass filter. The control performance is examined by changing the controller gain $G^{*}$ and phase shift $\phi$ of the output displacement of the baffle plate against the sensor signal.

\section{Modeling of Flexible Sheet and Control System}

\subsection{Mechanical model of flexible sheet}

As shown in Fig.1, the flexible sheet of length $L$ is divided into $n$ rigid bodies, which are connected each other by a rotational joint with the rotational spring $k_{\varphi}$ and rotational damper $c_{\varphi}$, which represent the bending stiffness and the damping of the sheet. The clamped point of the sheet is defined as the origin of the global coordinate system $o-x y$. The origin of the body coordinate system $o-x^{\prime} y^{\prime}$ of the $i$-th body is fixed at the gravity center of the body and the body angle expressed with the global coordinate system is defined by $\varphi_{i}$. The length, mass and moment of inertia of the body are $2 \ell, m$ and $J$, respectively. The flexible sheet is subjected to the fluid flow with flow velocity $U$ in the narrow passage and is excited by the dynamic fluid force resulting from the coupling between the sheet motion and fluid flow. The displacement $w$ of the sheet flutter at the downstream end is obtained numerically, and its sensor signal is sent to the controller to make a control signal.

\subsection{Constraint equation and equation of motion}

Generalized coordinate of the discrete system is defined as

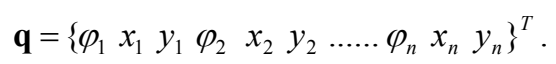

Kinematic constraint equation $\mathbf{C}^{\mathrm{K}}$ in the hinge between body $i-1$ and body $i$ is given by

$$
\mathbf{C}_{(i-1) i}^{K}=\left\{\begin{array}{c}
x_{i-1}-x_{i}-\ell \cos \varphi_{i-1}-\ell \cos \varphi_{i} \\
y_{i-1}-y_{i}-\ell \sin \varphi_{i-1}-\ell \sin \varphi_{i}
\end{array}\right\}=\mathbf{0}, \quad(i=1 \cdots n) .
$$

The constraint equation of all hinged joint of the body is written as follows form

$$
\mathbf{C}=\left\{\mathbf{C}_{12}^{K} \mathbf{C}_{23}^{K} \mathbf{C}_{34}^{K} \cdots \mathbf{C}_{(n-1) n}^{K}\right\}=\mathbf{0} .
$$

By differentiating the constraint equation $\mathbf{C}$ with respected to time, the velocity equation $\boldsymbol{v}$ is derived as

$$
\begin{aligned}
& \dot{\mathbf{C}} \equiv \mathbf{C}_{\mathbf{q}} \dot{\mathbf{q}}+\mathbf{C}_{t}=\mathbf{0}, \\
& \mathbf{C}_{\mathbf{q}} \dot{\mathbf{q}}=-\mathbf{C}_{t} \equiv \mathbf{v},
\end{aligned}
$$

where $\mathbf{C}_{\mathbf{q}}$ is Jacobian matrix of $\mathbf{C}$ with respected to $\mathbf{q}$, and is given by

$$
\mathbf{C}_{\mathbf{q}}=\frac{\partial \mathbf{C}}{\partial \mathbf{q}} \text {. }
$$

Subscript $\mathbf{q}$ and $t$ refer to the differentiation with respect to $\mathbf{q}$ and time.

Moreover, by differentiating the constraint equation $\mathbf{C}$ twice with respected to time, the acceleration equation $\gamma$ is derived as

$$
\begin{aligned}
& \ddot{\mathbf{C}} \equiv\left(\mathbf{C}_{\mathbf{q}} \dot{\mathbf{q}}\right)_{\mathbf{q}} \dot{\mathbf{q}}+\mathbf{C}_{\mathbf{q}} \dot{\mathbf{q}}+\mathbf{C}_{\mathbf{q}} \ddot{\mathbf{q}}+\mathbf{C}_{t \mathbf{q}} \dot{\mathbf{q}}+\mathbf{C}_{t t}=\mathbf{0}, \\
& \mathbf{C}_{\mathbf{q}} \ddot{\mathbf{q}}=-\left(\mathbf{C}_{\mathbf{q}} \dot{\mathbf{q}}\right)_{\mathbf{q}} \dot{\mathbf{q}}-2 \mathbf{C}_{\mathbf{q} t} \dot{\mathbf{q}}-\mathbf{C}_{t t} \equiv \boldsymbol{\gamma} .
\end{aligned}
$$


Mass matrix of the sheet is defined as

$$
\begin{aligned}
& \mathbf{M}_{i}=\operatorname{diag}\left[m_{i} m_{i} J_{i}\right](i=1 \cdots n), \\
& \mathbf{M}=\operatorname{diag}\left[\mathbf{M}_{1} \mathbf{M}_{2} \cdots \mathbf{M}_{n}\right] .
\end{aligned}
$$

Generalized force vector $\mathbf{Q}$ acting on the body is given as

$$
\begin{aligned}
& \mathbf{Q}_{i}=\mathbf{Q}_{i}^{A}+\mathbf{Q}_{i}^{F} \quad(i=1 \cdots n), \\
& \mathbf{Q}=\left\{\begin{array}{llll}
\mathbf{Q}_{1} & \mathbf{Q}_{2} & \cdots & \mathbf{Q}_{n}
\end{array}\right\}^{T},
\end{aligned}
$$

where $\mathbf{Q}_{i}^{F}$ is fluid force acting on the body $i$ and is derived in the following section, and $\mathbf{Q}_{i}^{A}$ is generalized force by the rotating spring and damper, which represent the bending stiffness and damping of the sheet. The generalized force $\mathbf{Q}_{i}^{A}$ is given as

$$
\begin{aligned}
\mathbf{Q}_{i}^{A}= & {\left[-k_{\varphi}\left(\varphi_{i}-\varphi_{i-1}\right)-k_{\varphi}\left(\varphi_{i}-\varphi_{i+1}\right)\right.} \\
& \left.-c_{\varphi}\left(\dot{\varphi}_{i}-\dot{\varphi}_{i-1}\right)-c_{\varphi}\left(\dot{\varphi}_{i}-\dot{\varphi}_{i+1}\right) m g 0\right]^{T} \quad(i=1 \cdots n),
\end{aligned}
$$

where $m g$ is gravity force, and $k_{\varphi}$ and $c_{\varphi}$ are spring and damping coefficients, respectively.

Using the Lagrange multipliers, the principle of virtual work in dynamics for a constrained system can be obtained as

$$
\begin{aligned}
& \boldsymbol{\lambda}=\left\{\begin{array}{llll}
\lambda_{1} & \lambda_{2} & \ldots . . & \lambda_{2 n}
\end{array}\right\}^{T}, \\
& {[\mathbf{M} \ddot{\mathbf{q}}-\mathbf{Q}]^{T} \delta \mathbf{q}+\boldsymbol{\lambda}^{T} \mathbf{C}_{\mathbf{q}} \delta \mathbf{q}=\left[\mathbf{M} \ddot{\mathbf{q}}-\mathbf{Q}+\mathbf{C}_{\mathbf{q}}^{T} \boldsymbol{\lambda}\right] \delta \mathbf{q}=\mathbf{0},}
\end{aligned}
$$

where $\lambda$ is Lagrange multipliers.

The equation of motion of the system with constraint is rewritten in the following augmented form

$$
\left[\begin{array}{cc}
\mathbf{M} & \mathbf{C}_{\mathbf{q}}^{T} \\
\mathbf{C}_{\mathbf{q}} & 0
\end{array}\right]\left\{\begin{array}{l}
\ddot{\mathbf{q}} \\
\boldsymbol{\lambda}
\end{array}\right\}=\left\{\begin{array}{l}
\mathbf{Q} \\
\boldsymbol{\gamma}
\end{array}\right\} .
$$

The equation of motion given by Eq.(16) is solved for all the Lagrange multipliers and the acceleration of the generalized coordinates that satisfy the constraint equations at acceleration level. By partitioning $\mathbf{q}$ into the independent coordinates part $\mathbf{q}_{\text {in }}$ and the dependent coordinates part $\mathbf{q}_{d}$, Eq.(16) can be reduced to the independent coordinate. By calculating the reduced independent equation of motion numerically, the dynamic behavior of the system can be obtained. Here, Runge-kutta method is applied in the computations.

\subsection{Fluid force acting on the body element}

The nonlinear fluid force acting on the body element is derived based on the numerical model developed by Kaneko and $\mathrm{Wu}$, in Ref.(5). The fluid flow is assumed to be 2-D, incompressible and viscous. The continuity and momentum equations of the leakage fluid flow in the narrow passage are described as

$$
\begin{aligned}
& \frac{\partial Q_{f}}{\partial x}+\frac{\partial h}{\partial t}=0 \\
& \frac{\partial Q_{f}}{\partial t}+\frac{\partial}{\partial x}\left(\frac{Q_{f}^{2}}{h}\right)=-\frac{h}{\rho_{f}} \frac{\partial P}{\partial x}-\frac{1}{4} \lambda_{f} \frac{Q_{f}{ }^{2}}{h^{2}},
\end{aligned}
$$

where $h$ is gap width of the passage, $Q_{f}, \rho_{f}$ and $\lambda_{f}$ are flow rate, fluid density and friction coefficient of the passage wall, respectively. The friction coefficient can be expressed as a function of Reynolds number as $\lambda_{f}=48 / \operatorname{Re}, \operatorname{Re}=\left|Q_{f}\right| / v$ in the case of 
laminar flow, and $\lambda_{f}=0.28 / \mathrm{Re}^{1 / 4}$ in the case of turbulent flow.

In the following, attention is paid to the fluid flow between the $i$-th body and the left wall of the passage, as shown Fig.2. In this figure, $x$-axis is defined along the center line of the passage, and the origin of the axis is set at the center of the body.

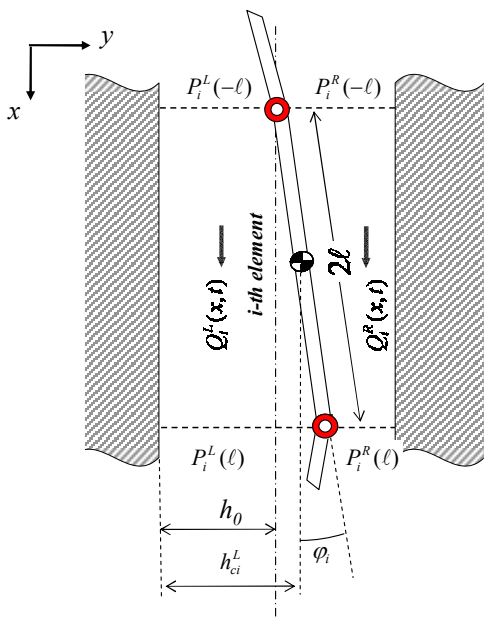

Fig.2 Motion of body element in the passage

By using the gap width $h_{c i}$ of the center of $i$-th body element and rotational angle of the body element $\varphi_{i}$, the gap width in the left passage can be written as

$$
h_{i}^{L}(x, t)=h_{c i}^{L}(t)+x \tan \varphi_{i}(t) \quad\left(i=i_{a}, \cdots i_{b}\right),
$$

where $L$ denotes the left passage, and $i$ denotes the $i$-th body element, and is defined from the inlet to outlet of the passage. Here, $i_{a}$ and $i_{b}$ denote the body element at the inlet and outlet of the passage, respectively.

By substituting Eq.(19) into Eq.(17) and integrating it with respect to $x$, flow rate is can be obtained as

$$
Q_{i}^{L}(x, t)=-\dot{h}_{c i}^{L} x-\frac{x^{2}}{2} \dot{\varphi}_{i} \sec ^{2} \varphi_{i}+C_{Q i}^{L}(t) \quad\left(i=i_{a}, \cdots i_{b}\right),
$$

where $C_{Q i}^{L}(t)$ is an integration constant as a function of time.

By substituting Eq.(19) and Eq.(20) into Eq.(18), pressure gradient is can be obtained as

$$
\frac{\partial P_{i}^{L}(x, t)}{\partial x}=W_{i}^{L}(x, t)-\frac{\rho_{f}}{h_{i}^{L}} \dot{C}_{Q i}^{L}(t) \quad\left(i=i_{a}, \cdots i_{b}\right),
$$

where $W_{i}^{L}(x, t)$ is given as

$$
\begin{aligned}
& W_{i}^{L}(x, t)=\frac{\rho_{f} \ddot{h}_{c i}^{L}}{h_{i}^{L}} x+\frac{\rho_{f}}{2 h_{i}^{L}} x^{2} \ddot{\varphi}_{i} \sec ^{2} \varphi_{i}+\frac{\rho_{f}}{h_{i}^{L}} x^{2} \dot{\varphi}_{i}^{2} \tan \varphi_{i} \sec ^{2} \varphi_{i} \\
& +\frac{2 \rho_{f} Q_{i}^{L} \dot{h}_{c i}^{L}}{h_{i}^{L^{2}}}+\frac{2 \rho_{f} Q_{i}^{L}}{h_{i}^{L^{2}}} x \dot{\varphi}_{i} \sec ^{2} \varphi_{i}+\frac{\rho_{f} Q_{i}^{L^{2}}}{h_{i}^{L^{3}}} \tan \varphi_{i}-\frac{\lambda_{f} \rho_{f}}{4 h_{i}^{L^{3}}} Q_{i}^{L}\left|Q_{i}^{L}\right| .
\end{aligned}
$$

The pressure and flow rate must be continuous between body $i$ and bodu $i+1$, the flowing equations are derived.

$$
\begin{aligned}
& P_{i-1}^{L}(\ell, t)=P_{i}^{L}(-\ell, t) \quad\left(i=i_{a}, \cdots i_{b}\right), \\
& Q_{i-1}^{L}(\ell, t)=Q_{i}^{L}(-\ell, t) \quad\left(i=i_{a}, \cdots i_{b}\right),
\end{aligned}
$$

Moreover, the boundary conditions at the inlet and outlet of the passage are given by 


$$
\begin{aligned}
& P_{i_{a}}^{L}(-\ell)=P_{a}-\xi_{a}^{L} \frac{\rho_{f}}{2} \frac{Q_{i_{a}}^{L}(-\ell, t)^{2}}{h_{i_{a}}^{L}(-\ell, t)^{2}}, \\
& P_{i_{b}}^{L}(\ell)=P_{b}+\xi_{b}^{L} \frac{\rho_{f}}{2} \frac{Q_{i_{b}}^{L}(\ell, t)^{2}}{h_{i_{b}}^{L}(\ell, t)^{2}},
\end{aligned}
$$

where $\xi_{a}$ and $\xi_{b}$ are pressure loss coefficients at the inlet and outlet of the passage, respectively, and $\xi_{a}$ and $\xi_{b}$ are dependent on the shape of the inlet and outlet. In this paper we assumed $\xi_{a}=1$ and $\xi_{b}=0$.

The fluid pressure in the left side passage is obtained as

$$
P_{i}^{L}(x, t)=P_{i_{a}}^{L}(-\ell, t)+\sum_{j=1}^{i-1} \int_{-\ell}^{\ell} \frac{\partial P_{j}^{L}(x, t)}{\partial x} d x+\int_{-\ell}^{x} \frac{\partial P_{i}^{L}(x, t)}{\partial x} d x \quad\left(i=i_{a} \cdots i_{b}\right),
$$

where, the fluid pressure out of the passage is given by

$$
P_{i}^{L}(x, t)=0 \quad\left(i=1 \cdots i_{a}-1, i=i_{b}+1 \cdots n\right) .
$$

Thus, the fluid force acting on the left side of the body element is obtained by

$$
\left\{\mathbf{F}^{L}\right\}=\frac{1}{2 \ell} \int_{-\ell}^{0}\left\{\mathbf{P}^{L}(x, t)\right\}(x+\ell) d x+\frac{1}{2 \ell} \int_{0}^{\ell}\left\{\mathbf{P}^{L}(x, t)\right\}(-x+\ell) d x,
$$

where

$$
\left\{\mathbf{F}^{L}\right\}=\left\{F_{1}^{L} F_{2}^{L} \cdots F_{n}^{L}\right\}^{T}, \quad\left\{\mathbf{P}^{L}(x, t)\right\}=\left\{P_{1}^{L}(x, t) P_{2}^{L}(x, t) \cdots P_{n}^{L}(x, t)\right\}^{T} .
$$

Similarly, the fluid force acting on the right side of the body element can be obtained by

$$
\left\{\mathbf{F}^{R}\right\}=\left\{F_{1}^{R} F_{2}^{R} \cdots F_{n}^{R}\right\}^{T} .
$$

Finally, the fluid force $\mathbf{Q}_{i}^{F}$ acting on the $i$-th body element can be obtained by the sum of Eq.(29) and Eq.(31).

\subsection{Active motion of the baffle plate actuator}

The fluid flow in the passage is actively controlled by the moving baffle-plate actuator set at the inlet of the passage to suppress the exciting fluid force. The motion of the baffle plate actuator is modeled as shown in Fig.3. By using the sensor signal $w$ with controller gain and phase shift, the output displacement of the baffle plate actuator is written as

$$
\left.\begin{array}{l}
u_{h}^{L}=G^{*} w\left(t-\frac{\phi}{\omega}\right), \quad \omega=2 \pi f_{f}, \\
u_{h}^{R}=-u_{h}^{L},
\end{array}\right\}
$$

where $G^{*}$ and $\phi$ are controller gain and phase shift, respectively. $f_{f}$ is the dominant frequency of the sheet flutter.

By using the output displacement of the baffle plate actuator $u_{h}^{R}$ and $u_{h}^{L}$, the varying gap width at the inlet of the passage can be written as

$$
\left.\begin{array}{l}
h_{i_{a}}^{L}(x, t)=h_{c i_{a}}^{L}(t)+x \tan \varphi_{i_{a}}(t)-u_{h}^{L}, \\
h_{i_{a}}^{R}(x, t)=h_{c i_{a}}^{R}(t)-x \tan \varphi_{i_{a}}(t)-u_{h}^{R} .
\end{array}\right\}
$$

From Eq.(32) and Eq.(25), it can be seen that the active motion of the baffle plate actuators has the effect on the pressure drop at the inlet, so that the fluid flow and fluid pressure in the passage can be controlled by the active motion of the baffle plate actuator. 

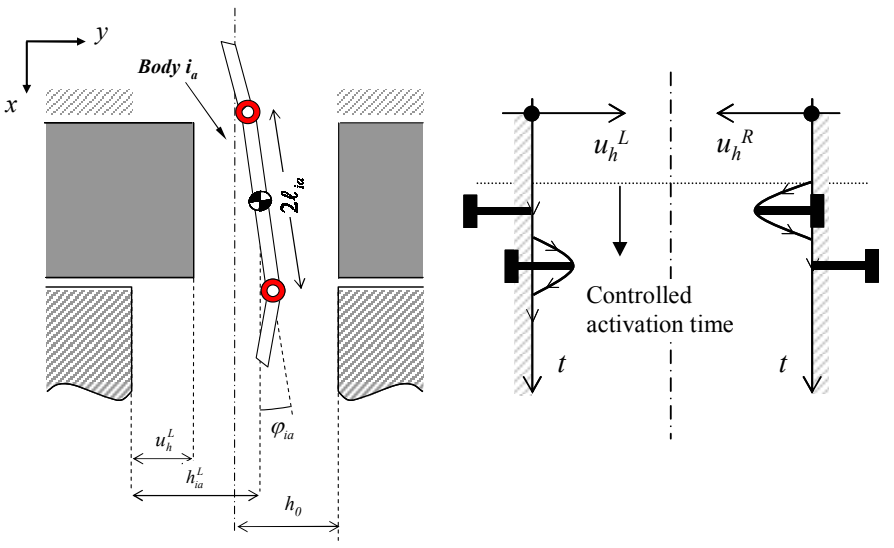

Fig.3 Active motion of baffle plate actuator

\section{Numerical Simulations}

\subsection{Analytical parameters}

We conducted numerical simulations to investigate the control performance of the proposed non-contact control device using the baffle plate actuators and the active feedback control method. The specification of the analytical system is shown in Table 1. The rotational spring constants, which are attached at joints connecting each body element, are determined to be close the natural frequencies of the discrete cantilever to those of the continuous cantilever.

Table.1 Specification of the analytical system

\begin{tabular}{|c|c|}
\hline Material of sheet & Bronze \\
\hline Fluid & Air \\
\hline Number of body $n$ & 12 \\
\hline Length of sheet $L$ & $0.29[\mathrm{~m}]$ \\
\hline Thickness of sheet $t_{h}$ & $0.3 \times 10^{-3}[\mathrm{~m}]$ \\
\hline Total mass of sheet $M_{s}$ & $0.768[\mathrm{~kg}]$ \\
\hline Young's modulus $E$ & $1.1 \times 10^{11}\left[\mathrm{~N} / \mathrm{m}^{2}\right]$ \\
\hline Density of sheet $\rho_{s}$ & $4.3 \times 10^{4}\left[\mathrm{~kg} / \mathrm{m}^{3}\right]$ \\
\hline Gap width $h$ & $2.0 \times 10^{-3}[\mathrm{~m}]$ \\
\hline Passage length $D$ & $0.156[\mathrm{~m}]$ \\
\hline
\end{tabular}

\subsection{Numerical results}

First, we examined the overall characteristics of the sheet flutter under the uncontrolled condition, in which the actuators are at rest. Fig.4 and Fig.5 show the time history and its spectrum of the vibration displacement at the sensor position, when the flow velocity is set at higher than the critical flow velocity. The variations of RMS (root-mean-square) of the vibration displacement and its dominant frequency with increasing flow velocity are shown in Fig.6. In the numerical simulations conducted in this study, it was observed that the flutter occurs in the second mode with a node at the outlet of the passage, and traveling-wave mode flutter was not observed. 
It can be seen that a violent flutter occurs to the flexible sheet over the critical flow velocity $U_{c r} \approx 8.6 \mathrm{~m} / \mathrm{sec}$. The amplitude of the sheet flutter increases exponentially with time, and then becomes constant in the limit-cycle state. The frequency of the flutter is about $7.0 \sim 8.0 \mathrm{~Hz}$ at the critical flow velocity and increases slightly with increasing flow velocity.

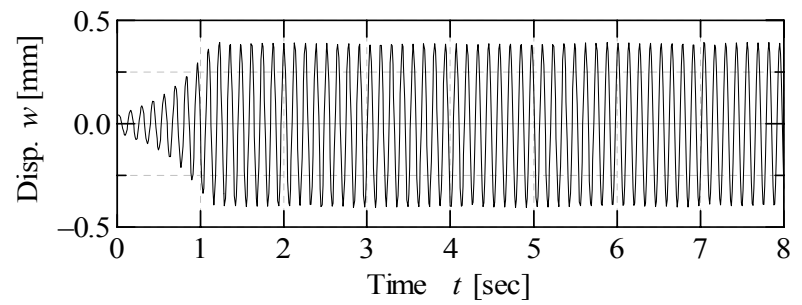

Fig.4 Time history of vibration displacement in the case of flow velocity $U=8.64 \mathrm{~m} / \mathrm{s}$

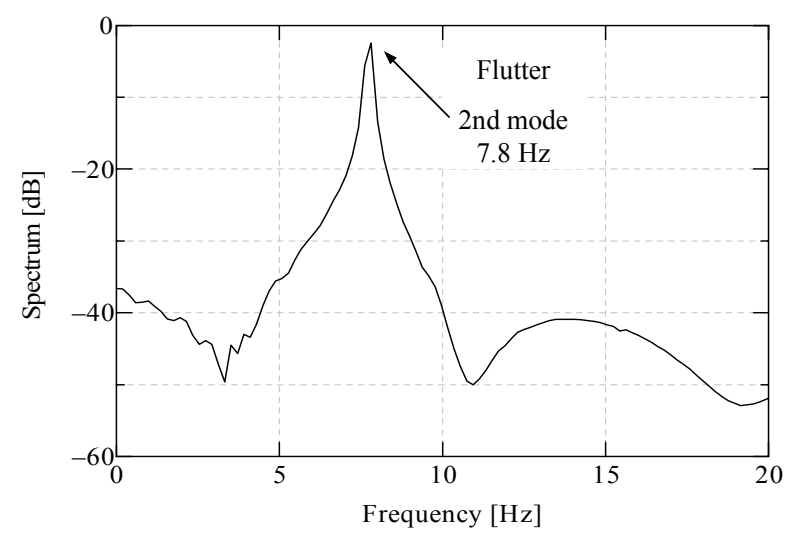

Fig.5 Spectrum of vibration displacement

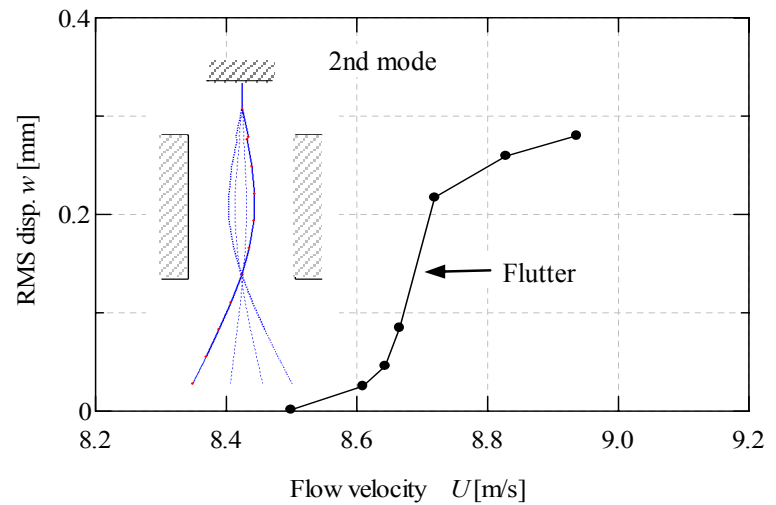

(a) RMS displacement

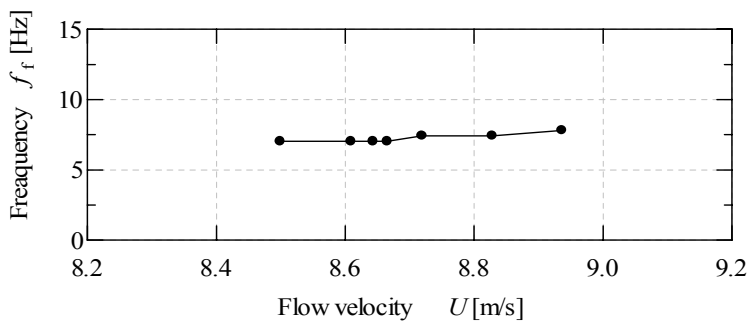

(b) Dominant frequency

Fig.6 Variation of vibration displacement and its frequency with increasing flow velocity; (a) RMS displacement, (b) dominant frequency 
Next, we examined the control performance of the proposed non-contact control device using the baffle plate actuators and the active feedback control method numerically. Typical time histories of the vibration displacement suppressed by the active control and the output displacements of the actuators are shown in Fig.7. These numerical results are in the case of the active feedback control $\phi=-30^{\circ}$ and $G^{*}=0.5$. It is seen that the sheet flutter is effectively suppressed by the active control, and the flutter amplitude decreases rapidly and exponentially by the active motion of the baffle plate actuator.

Moreover, in the case of $\phi=-30^{\circ}$ and $G^{*}=0.5$, time histories of the pressure fluctuations at point $\mathrm{A}$ (after inlet), $\mathrm{B}$ (in the middle), $\mathrm{C}$ (before outlet) in the passage are shown in Fig.8. It can be seen that the pressure fluctuation in the passage is suppressed by the active feedback control. Therefore, it is found that the pressure fluctuation caused by the fluid-structure interaction is suppressed by the active flow control, so that the flutter is effectively suppressed because the exciting fluid force resulting from the pressure fluctuation is suppressed.
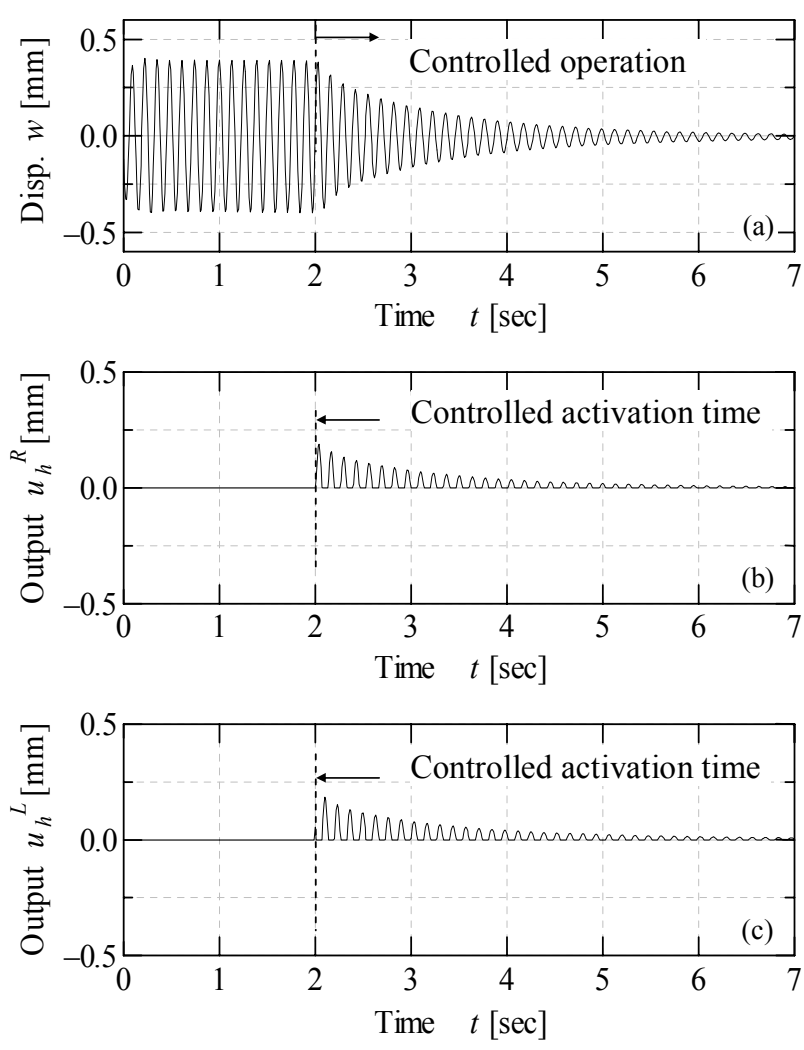

Fig.7 Time histories of stabilized system in the case of $\phi=-30^{\circ}, \mathrm{G}^{*}=0.5$; (a) vibration displacement $w$; (b), (c) output displacement of the actuator $u_{h}^{R}, u_{h}^{L}$ 

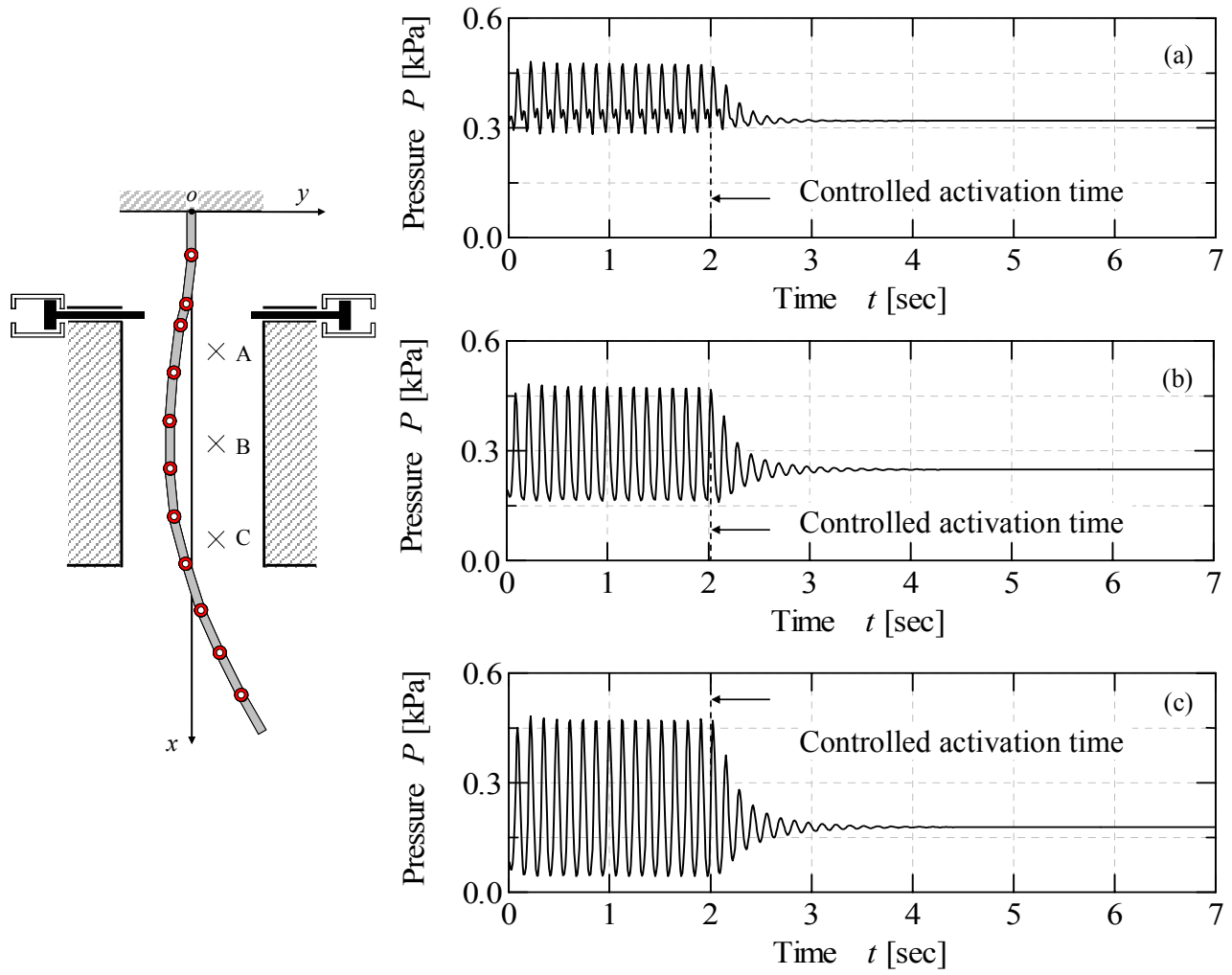

Fig.8 Time histories of pressure fluctuations in the case of $\phi=-30^{\circ}, \mathrm{G}^{*}=0.5$; at (a) Point A (after inlet), (b) Point B(in the middle) and (c) Point $\mathrm{C}$ (before outlet) in the passage

The variations of the time histories and those spectra of the flutter displacement suppressed by the active control are shown in Fig.9. These time histories were recorded with changing the controller gain $G^{*}$ from 0.3 to 0.8 , in the case of phase shift $\phi=-30^{\circ}$. For the case of $G^{*}=0.8$, sheet flutter is rapidly suppressed under the controlled operation. The stabilization effect by the active control increased with increasing the controller gain. The effect of the controller gain and phase shift on the RMS value ratio $w_{r m s-c} / w_{r m s}$ of the vibration displacement is shown in Fig.10. Here, the RMS of the vibration displacement under the controlled condition $w_{r m s-c}$ is normalized by that without control $w_{r m s}$.

It can be seen that, for the flow control at the inlet, when the phase shift is in the range of $-150^{\circ}<\phi<+30^{\circ}$, the sheet flutter is suppressed by the active control. The greatest stabilization effect was achieved when the phase shift was set in the range of $-60^{\circ}<\phi<0^{\circ}$. Moreover, it can be seen that the suppression effect becomes greater with increasing the controller gain. On the other hand, the system was destabilized by the active control when the phase-shift was set in the range of $+30^{\circ}<\phi<+180^{\circ}$. From these results, we can find that the performance of the active control is very sensitive to the phase-shift, and there is an optimum phase shift range to suppress the sheet flutter effectively. 

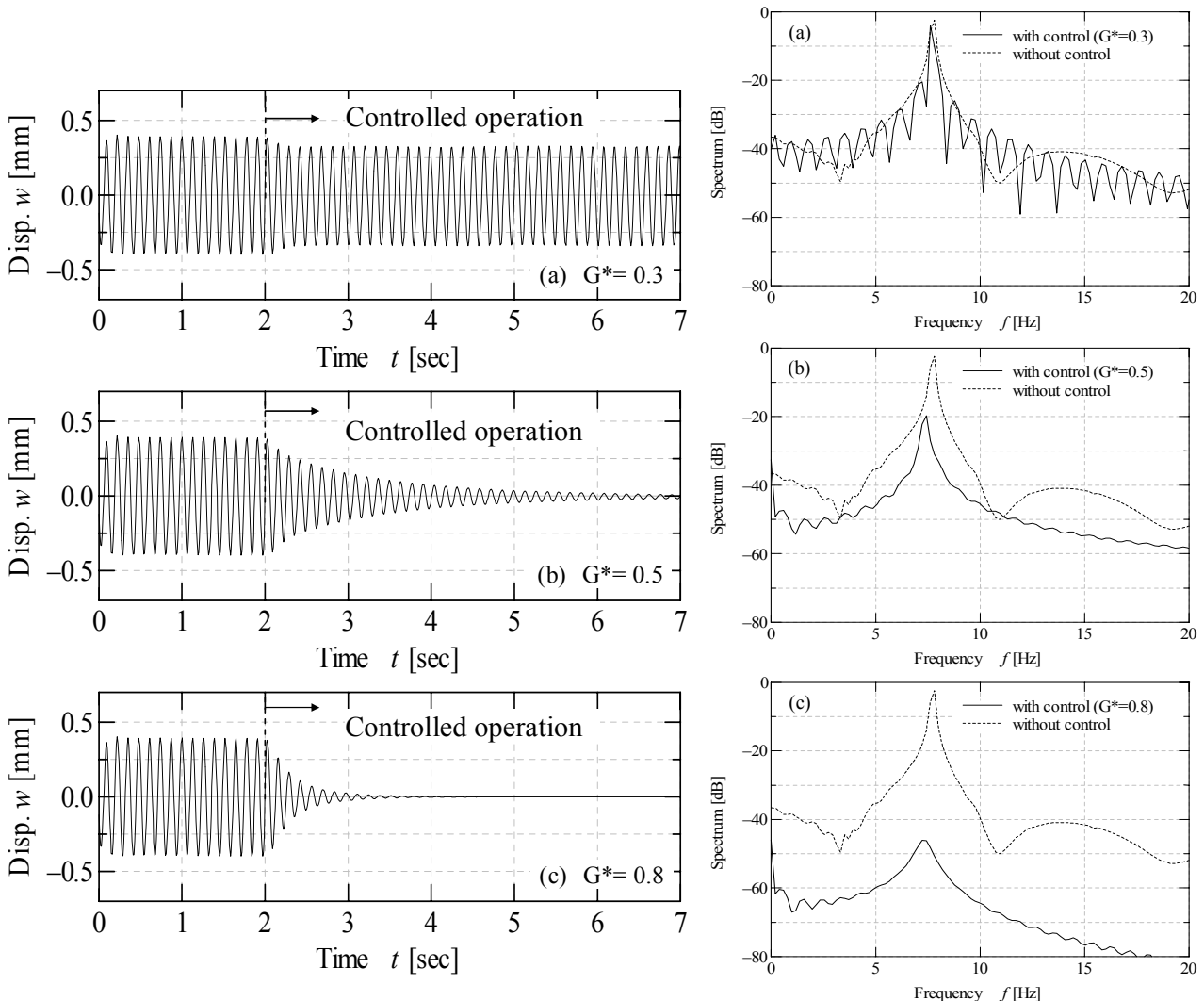

Fig.9 Time histories and spectra with changing controller gain $\mathrm{G}^{*}$ from (a) $\mathrm{G}^{*}=0.3$ to (c) $\mathrm{G}^{*}=0.8$ in case of $\phi=-30^{\circ}$

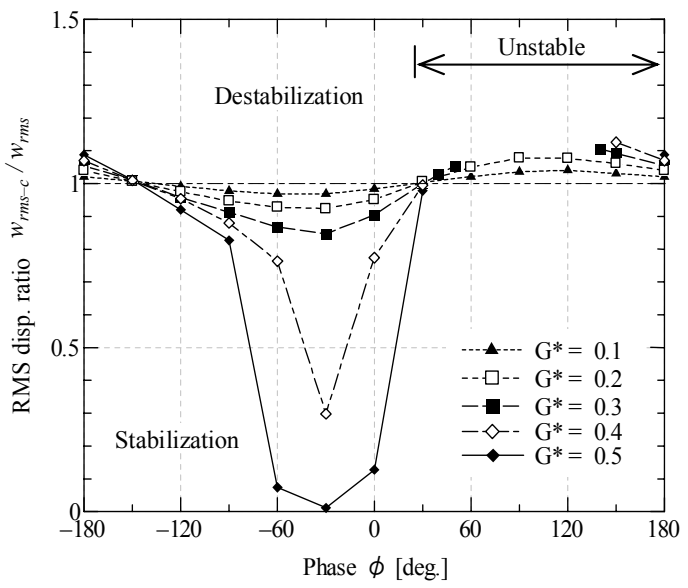

Fig.10 RMS ratio of vibration displacement $w_{r m s-c} / w_{r m s}$ with changing phase shift $\phi$ and controller gain $\mathrm{G}^{*}$

\section{Conclusions}

In this paper, we proposed a new non-contact control device and active feedback control technique, in which the moving baffle plate actuators are set at the inlet of the passage, to suppress the leakage-flow-induced sheet flutter in the passage. Then, we developed its numerical model based on multi-body dynamics formulation with considering the nonlinear fluid-structural interaction. The control performance is evaluated by the 
numerical simulations with changing the controller gain and phase shift. As a result, it was shown that the sheet flutter is effectively suppresses by the proposed control device and active feedback control technique because the exciting fluid pressure in the passage is suppressed by the active motion of the actuators. Moreover, it was indicated that the control performance is very sensitive to the phase shift, and there is the optimum phase shift range to suppress the sheet flutter effectively.

\section{References}

(1) Inada, F. and Hayama, S., A study on leakage-flow-induced vibrations: Fluid-dynamic forces acting on the walls of a one-dimensional, narrow passage, JSME Int. J. Series III, 31, pp.39-47, 1988.

(2) Nagakura, H. and Kaneko, S., The Stability of a Cantilever Beam Subjected to One-Dimensional Leakage Flow (in Japanese), JSME,58-546, C, pp.352-259, 1992.

(3) Yamaguchi, N., Yokota, K. and Tsujimoto, Y., Flutter Behavior of a Flexible Thin Sheet in High-Speed Flow: 1st Report (in Japanese), JSME, 65-632, B, pp.1224-1231, 1999.

(4) Yamaguchi, N., Sekiguchi, T., Yokota, K. and Tsujimoto, Y., Fluttering Behavior of a Flexible Thin Sheet in High-Speed Flow : 2nd Report (in Japanese), JSME, 65-632, B, pp.1232-1239, 1999.

(5) Kaneko, S. and Wu, X., Nonlinear Analysis of Sheet Flutter Based on Multibody Dynamics, Proc. of the First Asian Conference on Multibody Dynamics 2002, pp.486-493, 2002.

(6) Baz, A. and Ro, J., Active control of flow-induced vibrations of a flexible cylinder using direct velocity feedback, J. Sound and Vibration, 146-(1), pp.33-45, 1991.

(7) K. Roussopoulos, Feedback control of vortex shedding at low Reynolds numbers, J. Fluid Mech., 248, pp.267-296, 1993.

(8) Gunzburger, M.D. and Lee, H. C., Feedback control of Karman vortex shedding, J. Applied Mechanics, 63, pp.828-835, 1996.

(9) Huang, X.Y., Feedback control of vortex shedding from a circular cylinder, Experiments in Fluids, 20, pp.218-224, 1996.

(10) Ziada, S., Feedback control of globally unstable flows: Impinging shear flow, J. Fluids and Structures, 9, pp.907-923, 1995.

(11) Ziada, S., Ng, H. and Blake, C. E., Flow excited resonance of a confined shallow cavity in low Mach number flow and its control, J. Fluids and Structures, 18, pp.79-92, 2003.

(12) Huang, X.Y. and Weaver, D.S., 1991, On the active control of shear layer oscillations across a cavity in the presence of pipeline acoustic resonance, J. Fluids and Structures, 5, pp.207-219.

(13) Ffowcs Williams, J. E. and Huang, X. Y., Active stabilization of compressor surge, J. of Fluid Mech., 204, pp.245-2621, 1989.

(14) Kaneko, S. and Hirota, K., A study on active control of leakage flow induced vibrations, Proc. of the First Internal Conference on Motion and Vibration Control, pp.1135-1140, 1992.

(15) Watanabe, M. and Yamauchi, A., An Experimental Study on Active Control of Leakage- Flow-Induced Vibrations: Experiments for Translational One-DegreeFreedom System, CD-Rom Proceedings of ASME PVP Conference 2005, No.PVP2005-71221, 2005. 